Revue internationale P.M.E.

\title{
Adapter les outils de contrôle aux contextes et aux acteurs dans les PME : le cas exploratoire du tableau de bord achat d'un dirigeant
}

\section{To adapt the tools of control to the context and to the actors in SMEs : the exploratory case of a dashboard purchase of a leader}

\section{Adaptar los instrumentos de gestion a los contextos y a los actores en PyME : el caso de un cuadro de mando compra de un director}

Katia Dangereux, Philippe Chapellier et Fabienne Villesèque-Dubus

Volume 30, numéro 1, 2017

URI : https://id.erudit.org/iderudit/1039785ar

DOI : https://doi.org/10.7202/1039785ar

\section{Aller au sommaire du numéro}

Éditeur(s)

Editions EMS - In Quarto SARL

ISSN

0776-5436 (imprimé)

1918-9699 (numérique)

Découvrir la revue

Citer cet article

Dangereux, K., Chapellier, P. \& Villesèque-Dubus, F. (2017). Adapter les outils de contrôle aux contextes et aux acteurs dans les PME : le cas exploratoire du tableau de bord achat d'un dirigeant. Revue internationale P.M.E., 30(1), 27-56. https://doi.org/10.7202/1039785ar

\section{Résumé de l'article}

Ce travail a pour objectif de montrer que les systèmes de contrôle prennent du sens aux yeux des dirigeants de PME sous condition d'adaptation et que, dans certains cas, des systèmes de contrôle relativement proches de ceux susceptibles d'être observés dans les grandes organisations peuvent s'avérer adaptés aux besoins d'une PME. Pour ce faire, une étude a été réalisée au sein d'une PME d'une cinquantaine de salariés dans le cadre d'une observation participante. Nous proposons dans un premier temps une description d'un tableau de bord « achat » relativement élaboré, réactif, décentralisé, en lien avec la stratégie, en montrant comment il s'articule avec les autres outils de gestion de l'entreprise. Nous tentons ensuite d'identifier les caractéristiques organisationnelles, environnementales et individuelles permettant de comprendre la réussite perçue par le dirigeant de la mise en place de cet outil. 


\title{
Adapter les outils de contrôle aux contextes et aux acteurs dans les PME : le cas exploratoire du tableau de bord achat d'un dirigeant
}

\begin{abstract}
Katia DANGEREUX
Katia Dangereux est docteure en sciences de gestion, membre du laboratoire de recherche MRM et du LabEx Entreprendre et occupe un poste d'ATER à l'IAE de l'Université de Perpignan. Ses recherches portent sur la structuration des entreprises, via les outils de contrôle de gestion.

\author{
IAE de Perpignan - UPVD \\ 52, avenue Paul Alduy \\ 66860 PERPIGNAN CEDEX, France \\ katia.dangereux@univ-montp1.fr
}

Philippe CHAPELLIER

Philippe Chapellier est professeur des universités à Polytech Montpellier. Il est membre du laboratoire MRM et du LabEx Entreprendre. Ses travaux portent sur les outils de contrôle de gestion en PME et sur les transferts de connaissances entre professionnels comptables et dirigeants de PME.

Université de Montpellier

Polytech-Département IG

Place Eugène Bataillon

34095 MONTPELLIER, France

philippe.chapellier@umontpellier.fr

\section{Fabienne VILLESĖQUE-DUBUS}

Fabienne Villesèque-Dubus est professeure des universités en sciences de gestion à l'IAE de Perpignan et membre du laboratoire de recherche MRM. Ses recherches portent principalement sur les rôles instrumentaux, sociaux, stratégiques et politiques des outils de contrôle de gestion.

$$
\begin{array}{r}
\text { IAE de Perpignan - UPVD } \\
52, \text { avenue Paul Alduy } \\
66860 \text { PERPIGNAN CEDEX, France } \\
\text { fabienne.villeseque@univ-perp.fr }
\end{array}
$$

\section{RÉSUMÉ}

Ce travail a pour objectif de montrer que les systèmes de contrôle prennent du sens aux yeux des dirigeants de PME sous condition d'adaptation et que, dans certains cas, des systèmes de contrôle relativement proches de ceux susceptibles d'être observés dans les grandes organisations peuvent s'avérer adaptés aux besoins d'une PME. Pour ce faire, une étude a été réalisée au sein d'une PME d'une 
cinquantaine de salariés dans le cadre d'une observation participante. Nous proposons dans un premier temps une description d'un tableau de bord « achat » relativement élaboré, réactif, décentralisé, en lien avec la stratégie, en montrant comment il s'articule avec les autres outils de gestion de l'entreprise. Nous tentons ensuite d'identifier les caractéristiques organisationnelles, environnementales et individuelles permettant de comprendre la réussite perçue par le dirigeant de la mise en place de cet outil.

MOTS-CLÉS

Contrôle de gestion, Tableaux de bord achat. PME, Contingences

\title{
To adapt the tools of control to the context and to the actors in SMEs : the exploratory case of a dashboard purchase of a leader
}

\begin{abstract}
This article aims to show that management control systems make sense to SME's managers conditional to some adaptation and in some case, management control systems relatively close to those observed in big firms can be adapted to SMEs. To this end, a study has been driven in a SME of about fifty employees within the framework of a participating observation. Firstly, we propose to describe a "purchase " dashboard relatively elaborated, reactive, decentralized, linked with the strategy, showing how it is articulated with the others management tools of the organization. Then we attempt to identify organizational, environmental and individual characteristics allowing to understand the perceived achievement by the manager of the implementation of this management tool.
\end{abstract}

KEYWORDS

Management control, Purchase dashboards, SME, Contingencies

\section{Adaptar los instrumentos de gestion a los contextos y a los actores en PyME : el caso de un cuadro de mando compra de un director}

\section{RESUMEN}

Este trabajo tiene como objetivo mostrar que los sistemas de control toman todo su sentido ante los dirigentes de PyME a condición de adaptación y que, en ciertos casos, sistemas relativamente próximos de control de mismo que los susceptibles de ser observados en las grandes organizaciones, pueden revelarse adaptados a las necesidades de una PyME. Para hacerlo, un estudio ha sido realizado en el seno de una PyME de una cincuentena de salariados en el marco de una observación participante. Proponemos en primer tiempo una descripción de un cuadro de mando "compra " relativamente elaborado, reactivo y descentralizado, en lazo con la estrategia, mostrando cómo se articula con otros instrumentos de gestión de la empresa. Intentamos luego identificar las características organizativas, medioambientales e individuales para entender el éxito percibido por el dirigente de la colocación de este instrumento

\section{Palabras clave}

Control de gestión, Cuadro de mando compra, PyME, Contingencias 


\section{INTRODUCTION}

En 2013, la France comptait 3,2 millions de PME. Elles représentent 99,9 \% du tissu économique français, soit plus de $52 \%$ de l'emploi total et $49 \%$ de la valeur ajoutée ${ }^{1}$. Leur poids dans l'économie française est donc loin d'être négligeable. Ces entreprises tirent leur succès de leur réactivité, flexibilité, capacité à innover et savoir-faire unique. Les connaissances en gestion font parfois défaut à ces entrepreneurs ingénieux et créatifs (Chapellier, 2011 ; Zawadzki, 2011), mais la concurrence accrue et la mauvaise conjoncture économique semblent les pousser à chercher dans les outils de gestion un moyen d'améliorer leur performance afin de créer une valeur supérieure à celle de leurs concurrents ou de proposer des tarifs accessibles à des clients au budget parfois réduit (Oriot, Otley et Misiaszek, 2010 ; Oriot et Misiaszek, 2012 ; St-Pierre et Delisle, 2006). Oriot et Bergeron (2015, p. 175) expliquent ainsi que «les dirigeants de PME ont de plus en plus besoin d'outils de gestion leur permettant de représenter des situations complexes ». La création de masters en entrepreneuriat et PME ou les pratiques de conseil dans les petites structures expriment clairement ce besoin croissant des entrepreneurs d'aujourd'hui et de demain, de prescription des meilleures pratiques de gestion.

Parallèlement, les recherches sur le contrôle de gestion dans les PME ont connu un intérêt croissant ces deux dernières décennies permettant de pallier le déséquilibre entre les études relatives aux grandes entreprises et celles s'intéressant aux PME. Bon nombre de ces travaux soulignent la spécificité des PME par rapport à « leurs grandes sœurs ». Julien (2005) énumère précisément les traits qui, au-delà de la petite taille, définissent les spécificités du système de gestion de la PME : une personnalisation de la gestion, une gestion centralisée, un circuit décisionnel court, une faible spécialisation interne, une stratégie intuitive ou peu formalisée, et un système d'information peu complexe, peu organisé, basé essentiellement sur les contacts directs. Pour prendre leurs décisions, les dirigeants de PME s'appuieraient plutôt sur les sources d'informations externes qu'internes, sur les sources informelles que formelles, et sur les sources non financières que financières (Bescos et Mendoza, 1998; Beldi et Cheffi, 2005 ; Mintzberg, 2006; Vallerand, Berthelot et Morrill, 2008). En tant que sources formelles, internes et financières, les données de contrôle de gestion seraient plutôt mal adaptées aux besoins des dirigeants de PME et seraient reléguées au second plan par ceux-ci.

Le parti pris de la spécificité n'est cependant pas partagé par tous les chercheurs (Courrent et Villesèque-Dubus, 2008). Un second paradigme relatif aux PME souligne la diversité de leurs pratiques. Tel est le cas par exemple de la théorie du cycle de vie (Moores et Yuen, 2001) évoquant l'existence de plusieurs étapes de développement à l'origine de pratiques, notamment de contrôle, bien distinctes entre les PME. Il y aurait alors non pas " une PME » au visage unique et universel, mais « des PME » diverses qui subissent des mutations au gré de leur avancement et positionnement dans le temps. Tel est le cas également de la théorie de la contingence qui affirme que des facteurs de contingence interagissent pour créer des situations uniques et atypiques rendant toutes tentatives d'uniformisation des organisations inutiles. Les caractéristiques des systèmes de contrôle des PME varieraient en fonction des

$1 \quad$ Annexes du document budgétaire Effort financier de l'État en faveur des petites et moyennes entreprises publié dans le projet de loi de finances 2014. 
caractéristiques structurelles et environnementales de l'entreprise et du profil des acteurs en présence (Rowe, Fernandez et Picory, 1994 ; Lavigne, 2002 ; Affès et Chabchoub, 2007). Torrès (2004) va même au-delà et affirme qu'il existe des PME « contre-nature » susceptibles de ne pas, ou ne plus, être spécifiques au point de ressembler aux grandes.

Les recherches empiriques réalisées sur le thème des systèmes de contrôle en PME aboutissent donc à des conclusions plutôt contrastées. Certains auteurs affirment que les dirigeants des entreprises les plus petites disposent de systèmes de contrôle peu complexes et peu formalisés (Davila, 2005 ; Zawadzki, 2011). D’autres soulignent que cette vision ne correspond pas à la réalité de l'ensemble des pratiques en PME et relèvent que les caractéristiques des systèmes de contrôle varient d'une PME à l'autre (Affès et Chabchoub, 2007 ; Bonache, Chapellier, Ben Hamadi et Mohammed, 2015) pouvant même, dans certains cas s'avérer proches de celles des grandes (Torrès, 2004).

L'absence de consensus rend toute tentative de prescription ou du moins d'enseignement des «bonnes pratiques » de contrôle de gestion en contexte PME difficile et périlleuse. Il nous semble donc important de poursuivre les investigations sur cette question afin de savoir quel discours adopter face à des entrepreneurs en quête de structuration. Faut-il prescrire un modèle de contrôle de gestion unique, simple, rudimentaire et peu formalisé ? Doit-on au contraire faire passer un message d'appropriation des outils de contrôle au regard de la structure qui les accueille?

Dans la lignée des recherches portant sur la diversité des pratiques de contrôle de gestion observée dans les PME, nous pensons que, sous l'effet de diverses contingences, les systèmes de contrôle de gestion diffèrent d'une PME à l'autre au point d'apparaître pour certaines d'entre elles relativement formalisés et élaborés. Néanmoins, nous partageons le point de vue de Condor et Rebut (2008) selon lequel les recherches sur cette question, pour l'essentiel quantitatives, mettent en évidence l'existence de nombreux facteurs de contingence sans véritablement les approfondir. À l'instar de Meyssonnier et Zawadzki (2008), Nobre et Zawadzki (2013), et Oriot et Misiaszek (2012), nous souhaitons donc analyser les pratiques de contrôle de gestion dans les PME par le biais d'une méthodologie de type qualitatif au travers de la méthode du cas unique. Plus précisément, une étude a été menée au sein d'une PME d'une cinquantaine de salariés dans le cadre d'une observation participante réalisée par l'un des coauteurs sur une période de cinq mois. Ce type d'approche permet une connaissance plus en profondeur de l'entreprise et des acteurs en présence et semble particulièrement approprié pour tenter de comprendre l'influence des facteurs de contingence sur les pratiques de contrôle de gestion dans les PME, voire pour faire émerger de nouveaux facteurs de contingence encore inexplorés par les études quantitatives.

Dans la lignée de l'étude de Germain (2001), dont le but était de déterminer dans quelle mesure et sous quelles conditions les tableaux de bord pouvaient être adaptés au contexte "PME », deux objectifs sont attachés à ce travail :

1. Proposer une description d'un tableau de bord « achat » mis en place dans cette PME et observer comment il s'articule avec les outils de gestion existants, afin de montrer qu'un outil de contrôle relativement élaboré, voire proche de ceux susceptibles d'être observés dans les grandes organisations, peut s'avérer adapté aux besoins d'une PME ; 
2. Identifier les caractéristiques organisationnelles, environnementales et individuelles permettant de comprendre la réussite perçue par le dirigeant de la mise en place de cet outil.

Après avoir présenté une revue de la littérature et indiqué la problématique de la recherche, nous exposons la méthodologie mise en œuvre et les résultats obtenus. Ceux-ci sont ensuite discutés dans une dernière partie.

\section{REVUE DE LITTÉRATURE ET PROBLÉMATIQUE}

L'état de l'art sur la question du contrôle de gestion en contexte PME fait émerger deux grands paradigmes. Pour certains auteurs (Raymond, 1995 ; Torrès, 2000 ; Julien, 2005), le système de contrôle à l'œuvre au sein des PME est spécifique à celles-ci. Les pratiques dans ce type d'entreprises seraient relativement homogènes et ferait apparaître un système de contrôle simple et informel (1.1.). Pour d'autres (Van Caillie, 2003 ; Germain, 2004), le système de contrôle n'est pas spécifique à la catégorie des $\mathrm{PME}$, mais propre à chaque PME. Ces derniers soulignent ainsi, la grande diversité des systèmes de contrôle observables en PME (1.2.).

\subsection{Un système de contrôle homogène aux PME}

\subsubsection{LA THÉORIE DE LA SPÉCIFICITÉ DES PME}

Les tenants de ce courant de recherche tendent à considérer que les PME sont dotées de caractéristiques similaires et constituent de ce point de vue un groupe homogène, par opposition à celui des grandes entreprises. Raymond (1995) note la spécificité du système d'information, la spécificité psychosociologique, la spécificité environnementale et la spécificité décisionnelle et organisationnelle de la PME. Julien (2005) souligne quant à lui la présence d'un système d'information simple, d'une forte polyvalence et interchangeabilité entre les membres de l'organisation, d'une stratégie intuitive et d'un système de gestion centralisé. Torrès (2000) met en exergue le rôle et l'importance de la proximité dans le contexte PME.

Ainsi, avec certaines nuances toutefois, ces auteurs soutiennent la thèse de la spécificité des modes de fonctionnement et de l'uniformité de la «famille » PME. Les pratiques de contrôle de gestion en PME seraient de ce fait relativement homogènes. Elles se caractériseraient notamment par la simplicité et la faible formalisation du système de contrôle.

\subsubsection{UN SYSTÈME DE CONTRÔLE SIMPLE ET INFORMEL}

Une partie de la littérature laisse ainsi apparaître que bon nombre de dirigeants d'entreprises de taille petite ou moyenne ne disposent que d'outils de gestion embryonnaires et note l'attraction des dirigeants de PME pour les médias les plus informels et pour les informations verbales (MacMahon et Holmes, 1991 ; Davila, 2005 ; Zawadzki, 2011). Les chefs de PME auraient, pour prendre leurs décisions, le plus souvent recours à leurs seuls jugements, intuitions et expériences et auraient, pour la plupart, une propension naturelle à penser qu'ils 
sont capables de gérer leur affaire seuls, sans autre système d'information de gestion que celui constitué par quelques données comptables qu’ils jugent essentielles.

La comptabilité en général, et le contrôle de gestion en particulier, seraient mal adaptés aux besoins des dirigeants de PME pour deux raisons essentielles. La première concerne les difficultés du couplage entre des processus de décision peu ou pas structurés et l'usage d'outils structurants par nature. La seconde est liée à ce que les outils comptables sous-tendent des types de modèles de gestion bien structurés, leur vocation initiale serait donc celle d'une utilisation par des décideurs situés dans des environnements relativement stables et générateurs d'événements plutôt répétitifs. Or, les PME relèvent le plus souvent d'une logique du petit nombre d'événements, nécessairement peu répétitifs, peu réguliers (Chapellier, 2011).

Ainsi, le système de contrôle des PME serait relativement simple et principalement constitué d'indicateurs financiers classiques comme le chiffre d'affaires, la marge, la trésorerie, quelques calculs des coûts, et quelques ratios de rentabilité. Le contrôle s'exercerait de façon informelle, notamment à travers la culture d'entreprise, et serait assuré par le partage des valeurs véhiculées par le dirigeant et intériorisées par tous les membres de l'organisation. Les systèmes de contrôle en PME se caractériseraient donc par une faible formalisation et une grande simplicité (Whitley, 1999).

Mais un autre pan de la littérature montre que les pratiques en la matière varient fortement d'une PME à l'autre.

\subsection{Un système de contrôle adapté à chaque PME}

Tout un pan de la littérature montre que la pluralité des caractéristiques des PME engendre une diversité des pratiques en général, et des systèmes de contrôle en particulier (Rowe, Fernandez et Picory, 1994 ; Lavigne, 2002 ; Affès et Chabchoub, 2007). Comme le souligne Van Caillie (2003), le paradigme de la spécificité gomme pour partie la multiplicité quasi infinie des caractéristiques de chaque PME et ne retranscrit ainsi que partiellement la réalité des pratiques en PME.

\subsubsection{Théorie De LA CONTINGENCE ET CONTRÔLE DE GESTION EN PME}

Plusieurs recherches ont montré l'existence de liens entre les caractéristiques structurelles et contextuelles de la PME et son système de contrôle. Germain (2004, p. 127) explique par exemple que : "les tableaux de bord sont, au même titre que les autres outils de contrôle de gestion, soumis à des contingences qui les amènent à adopter des formes différentes dépendantes $d u$ contexte organisationnel dans lequel ils s'insèrent ». Le facteur " taille " a souvent été mis en avant par les auteurs (Nobre, 2001 ; Chenhall, 2003). Il permet de relativiser l'idée selon laquelle les PME constitueraient un bloc homogène face à un second, celui des grandes entreprises. Dans quelle mesure est-il en effet pertinent d'envisager une homogénéité des pratiques entre une PME qui compte 10 salariés, une autre qui en compte une centaine et une troisième qui se rapproche des 250 salariés ? Nobre (2001) relève l'existence de deux stades du contrôle de gestion en fonction de la taille de la PME : le premier concerne les entreprises de moins de 100 salariés, le second regroupe celles qui comptent entre 100 et 250 salariés. Ce résultat rejoint ceux des 
auteurs de la théorie du cycle de vie de la firme (Moores et Yuen, 2001) qui relèvent l'existence de différents stades de développement des organisations induisant des conceptions différentes des systèmes de contrôle à l'œuvre au cours des étapes du cycle de vie. Les modes de gestion informels de la petite entreprise laisseraient place à un système de plus en plus formalisé au fur et à mesure que l'entreprise croît (Flamholtz, 1986) se rapprochant ainsi des systèmes observés dans les grandes entreprises. D'autres auteurs ont par ailleurs montré l'existence de liens entre le système de contrôle et la structure de propriété (Lavigne, 2002 ; Affès et Chabchoub, 2007 ; Cheffi et Nekhili, 2011), le niveau d'endettement (Coker et Hayes, 1992 ; Lavigne et St-Pierre, 2002), l'exportation (Lavigne, 2002 ; Vallerand, Berthelot et Morrill, 2008), le type de stratégie (Chenhall, 2003 ; Jänkälä, 2007 ; Boulianne, 2007), ou l'incertitude perçue de l'environnement (Haldma et Lääts, 2002 ; Germain, 2004 ; Abdel-Kader et Luther, 2008).

Mais la théorie de la contingence structurelle néglige l'influence des acteurs. D’autres auteurs (Macintosh, 1985 ; Affès et Chabchoub, 2007 ; Santin et Van Caillie, 2008 ; Chapellier et Ben Hamadi, 2012) ont proposé pour cette raison d'élargir le cadre de recherche sur les systèmes de contrôle à la théorie de la contingence comportementale. Dans cette perspective, les caractéristiques individuelles de chaque acteur viennent modérer l'aspect normatif des variables structurelles. Un individu peut être actif par rapport à la situation et non contraint par elle. Ainsi, et plus précisément, les caractéristiques organisationnelles et environnementales peuvent certes, dans une certaine mesure, limiter et déterminer les choix de l'acteur, mais ce dernier n'en reste pas moins libre et doué d'intentionnalité. Il dispose d'une marge de liberté (Crozier et Friedberg, 1977). Les facteurs de contingence relatifs aux acteurs en PME retenus par les auteurs tournent le plus souvent autour de l'acteur central de la PME : son dirigeant. Le profil du dirigeant (formation, expérience, histoire, culture...) semble jouer un rôle très particulier qui fait la particularité de ce type d'organisation : "comprendre la PME, c'est tout d'abord découvrir le profil et percer les motivations de son propriétaire-dirigeant " (Raymond, Blili et ElAlami, 2004, p. 58). Des caractéristiques telles que son niveau et son type de formation (Saboly, 1994 ; Chapellier, 2003), son expérience (Chapellier, 2003), son degré de participation au capital (Lavigne, 2002 ; Ngongang, 2007), impacteraient son système de contrôle.

Ce pan de la littérature soutient ainsi que les pratiques de gestion en PME sont différenciées, car adaptées au contexte et aux profils des acteurs. Elles varieraient en fonction de facteurs de contingence organisationnels et environnementaux d'une part, individuels et comportementaux d'autre part (Raymond et Bergeron, 2008).

\subsubsection{Un SYSTÈmE DE CONTRÔle AdAPTÉ AU CONTEXTE ET AU PROFIL DES ACTEURS DANS CHAQUE PME}

Chapellier (2011, p. 32) affirme que « la vision réductrice du système d'information comptable, orientée vers la production des seuls documents obligatoires, dans des délais longs, et dans le seul but de satisfaire aux obligations imposées par l'administration fiscale, ne correspond pas à la réalité d'une grande majorité des $P M E »$.

Plusieurs auteurs mettent aussi en lumière la présence dans les PME de systèmes de contrôle formalisés à des degrés divers, via la présence d'outils de gestion comptable :

- Nobre (2001) constate que "la procédure budgétaire et le calcul d'écart constituent des démarches très courantes, puisqu'elles sont mises en cuvre dans respectivement $70 \%$ et $81 \%$ des 
PME de l'échantillon» (p. 24) et que les tableaux de bord «sont largement répandus, puisque seul $5 \%$ de l'effectif ne les utilisent pas» (p. 22);

- Chapellier, Mohammed et Teller (2013) notent que des situations comptables intermédiaires sont formalisées dans la plupart des 91 PME de leur échantillon, qu'une majorité d'entre elles dispose d'un système de calcul de coûts et d'un tableau de bord. Ils expliquent en revanche que les données budgétaires et les calculs d'écarts semblent moins présents ;

- Germain (2001) révèle que « les tableaux de bord des entreprises de l'échantillon sont relativement complexes sur le plan technique» (p. 9);

- Bergeron (2002) montre qu'une grande majorité de PME dispose de systèmes multidimensionnels de mesure de la performance. Elle note que $72 \%$, $78 \%$ et $83 \%$ des PME de son échantillon sont respectivement dotées de mesures relatives à l'innovation, à la clientèle et à la qualité. Elle en conclut que des indicateurs de performance financiers, mais aussi non financiers, en adéquation avec les objectifs stratégiques, sont présents dans les PME.

L'ensemble de ces recherches met ainsi en lumière qu'en contexte PME, le contrôle peut être formalisé via la présence d'outils de gestion comptable. Elles soulignent que ces outils ne sont pas forcément rudimentaires, et peuvent parfois constituer de véritables outils de pilotage axés sur la stratégie et prendre en compte l'aspect multidimensionnel de la performance (Oriot et Bergeron, 2012). Ces recherches montrent que les systèmes de contrôle en PME sont diversifiés. Chapellier, Mohammed et Teller (2013, p. 68) expliquent ainsi qu'il n'existe pas de norme dictant le degré de formalisation adéquat du système de contrôle en PME : "L'enjeu pour le dirigeant est de trouver le bon équilibre sur une sorte de balancier entre l'absence et l'excès de formalisation comptable... Si certains dirigeants de PME disposent d'un système de contrôle simple et peu formalisé, d'autres élaborent à fréquence régulière des données de contrôle diversifiées et détaillées ». Oriot et Misiaszek (2012, p. 40) invitent ainsi «les dirigeants de PME à construire des tableaux de bord stratégiques taillés sur mesure par rapport à leur expérience, leur contexte spécifique et l'évolution de leurs représentations cognitives".

Dans la continuité de la théorie de la contingence, nous pensons que les caractéristiques des systèmes de contrôle en PME varient en fonction de variables structurelles et comportementales pouvant aboutir, selon le contexte et le profil des acteurs, à la mise en œuvre et à l'utilisation de systèmes de contrôle relativement élaborés, réactifs, décentralisés, en lien avec la stratégie, proches de ceux susceptibles d'être observés dans les grandes entreprises.

Nous optons ainsi dans cet article pour le paradigme de la diversité des PME. Nous cherchons à identifier, au sein de cette diversité, ce qui peut être propre à chaque PME en explorant, via une étude de cas unique, les variables susceptibles d'influencer leur système de contrôle.

Deux objectifs sont ainsi attachés à cette étude :

- proposer une description d'un tableau de bord «achat» mis en place dans une PME et indiquer comment il s'articule avec les outils de gestion existants, afin de montrer qu'un outil de contrôle relativement élaboré, voire proche de ceux susceptibles d'être observés dans les grandes organisations, peut s'avérer adapté aux besoins d'une PME ;

- identifier les caractéristiques organisationnelles, environnementales et individuelles permettant de comprendre la réussite perçue par le dirigeant de la mise en place de cet outil. 


\section{MÉTHODOLOGIE ET DESCRIPTION DU TERRAIN DE RECHERCHE}

Pour réaliser cette recherche, nous avons choisi d'étudier une PME commerciale languedocienne dans le cadre d'une observation participante réalisée par l'un des coauteurs. Une série d'entretiens a permis de compléter cette analyse.

\subsection{Une méthode basée sur plusieurs modes de collecte de données}

Mener une série d'entretiens en parallèle de l'observation participante présente l'intérêt de collecter deux types de données complémentaires. Les données factuelles de l'observation participante sont ainsi complétées par les données discursives des différents entretiens réalisés.

\subsubsection{L'OBSERVATION PARTICIPANTE}

L'observation participante, d'une durée de cinq mois (du 01/04/2012 au 31/08/2012) a été effectuée, dans le cadre de la réalisation d'un mémoire de stage de master recherche, au sein du service achat de l'entreprise. La forte volonté de la direction et de la responsable achat de se doter d'un instrument de suivi et de pilotage les a conduits à dessiner les contours de ce qui aura été la mission principale de cette observation participante : la conception et la réalisation, en collaboration avec cette responsable, d'un tableau de bord achat. Un autre des chercheurs, associé à cette étude, a participé à plusieurs réunions avec le dirigeant, la responsable achat, et le directeur financier, ainsi qu'aux entretiens réalisés avec le dirigeant.

Le chercheur peut ici être qualifié «d'observateur qui participe» (Thiétart, 2014) : sa participation à la vie de l'organisation et à la mise en place de l'outil a été importante, mais son rôle de chercheur était clairement défini auprès des sujets sources. Cette situation aurait pu provoquer des résistances, mais tel n'a pas été le cas. Cette méthode n'est pas sans limites. Mucchielli (1988) dénonce les biais perceptuels et interprétatifs qu'un chercheur introduit dans l'acte d'observation. Du fait de ses centres d'intérêt du moment, de son expérience, et de ses choix intellectuels, le chercheur peut inconsciemment « ne voir que ce qui lui convient, n'entendre que ce qu'il veut bien entendre, et oublier ce qui lui est désagréable " (Mucchielli, 1988, p. 14). Sa présence influe par ailleurs sur les comportements et actions des acteurs observés et inversement. Nous sommes conscients de ces limites, mais la réalisation d'une observation participante nous est apparue adaptée pour répondre aux objectifs de l'étude. Cette méthode permet la saisie de logiques de situations liées à un temps et un contexte spécifique et «donne plus de relief aux résultats » (Hlady-Rispal, 2002). Elle permet de ne pas réduire le phénomène observé à ce que les acteurs peuvent en dire. Cette observation pourrait ainsi permettre de mieux comprendre pourquoi des petites structures ressentent la nécessité de développer de nouveaux instruments de gestion, quels sont les éléments déclencheurs, facilitateurs, mais également les freins à cette mise en place, et d'établir un lien entre les variables de contingence et l'outil implémenté, sa nature, et son degré d'élaboration.

Cette observation a été facilitée par la forte interdépendance des différents services entre eux et par la place centrale du service achat au sein du réseau interne, mais également vis-à-vis de 
l'extérieur (avec les fournisseurs et les prestataires de transport). Le service achat est en effet au cœur de problématiques très variées impliquant la quasi-totalité des autres fonctions de l'entreprise. Sa responsable est ainsi une interlocutrice centrale qui participe aux réunions entre la direction et les responsables commerciaux (puisque c'est elle qui décide, au regard des informations qui lui sont transmises, de la composition du portefeuille de produits et de gammes), le directeur financier (notamment sur des questions relatives à la gestion des stocks et la trésorerie), mais aussi avec les responsables des services marketing et logistique.

L'observation étant participante, la place du chercheur est particulière. Plus précisément, ce dernier fut au cœur du processus de mise en place du nouveau tableau de bord achat. Son rôle principal était d'assurer l'intermédiation entre les différents acteurs concernés par l'outil. Il recueillait les indicateurs utilisés par la chargée des approvisionnements, les deux employées achat, la responsable achat et le directeur financier, et discutait avec les acteurs des informations prioritaires pour ensuite sélectionner un nombre restreint d'indicateurs jugés pertinent au regard des objectifs fixés par le dirigeant. Ces indicateurs devaient répondre aux besoins d'information pour la gestion du service achat d'une part et pour la gestion de la chaîne logistique d'autre part. In fine, ces cinq acteurs ainsi que le chef produit, la chargée de catalogue et le responsable marketing devaient pouvoir se retrouver autour de cet outil pour résoudre des problématiques communes.

Les interactions quotidiennes entre le chercheur, la responsable achat et les deux employées du service « achats » ont largement enrichi le recueil des données. Le chercheur a également beaucoup interagi avec l'ingénieur informatique chargé de la récupération des données historiques afin de procéder à l'automatisation du tableau de bord. Les échanges portaient sur la question du paramétrage, la fréquence d'édition et les variables à mobiliser dans les calculs. Le chercheur a ainsi joué le rôle d'interface entre les besoins d'information pour l'ensemble des acteurs impliqués dans la chaîne logistique et les compétences techniques de l'ingénieur informatique pour automatiser le calcul des différents indicateurs retenus.

La place du chercheur dans la PME a aussi permis l'accès à divers documents d'entreprise. Parmi les documents analysés, on trouve les documents comptables officiels, l'ancien tableau de bord achat qui avait été rapidement abandonné, des documents présentés lors de réunions entre la direction, les responsables achat, logistique, informatique et financier et leurs collaborateurs et des procédures relatives au fonctionnement du service achat. Les sources documentaires nous ont permis de compléter les observations et de rapprocher les observations et les discours, des données chiffrées et des procédures prévues.

Le chercheur a enfin pu assister à la très grande majorité des réunions et comités de pilotage regroupant les différents acteurs, ce qui nous a offert un regard panoramique sur l'ensemble de l'entreprise.

Au final, les interactions et discussions quotidiennes réalisées tout au long des cinq mois de l'observation entre le chercheur et l'ensemble des parties prenantes, la participation du chercheur aux réunions et comités de pilotage et l'analyse documentaire ont constitué une base importante du recueil des données. En complément, plusieurs entretiens ont été réalisés à différents moments de l'étude. 


\subsubsection{LES ENTRETIENS}

Trois entretiens non directifs (auxquels un autre chercheur associé à l'étude a participé) avec les deux acteurs majeurs de ce tableau de bord, à savoir le dirigeant et la responsable achat, ont été réalisés au début, au milieu et un mois avant la fin de la période de mise en place de l'outil, afin de mieux cerner l'usage et les fonctionnalités attendus du tableau de bord.

Un entretien a également été réalisé au début du stage avec les deux employées du service achat, dans le but de comprendre leurs tâches respectives au sein du service, de déterminer les enjeux majeurs du service pour l'entreprise, d'identifier leurs différents interlocuteurs et les liens avec les autres services et avec les parties prenantes externes (les fournisseurs et prestataires logistiques).

Enfin, un an après la mise en place de l'outil, un entretien semi-directif d'une durée d'une heure et trente minutes a été réalisé à des fins de validation par les chercheurs avec le dirigeant de Phonepro. Cet entretien nous a permis de connaître le devenir du tableau de bord achat et d'apporter des éléments de réponse à des questions telles que : le tableau de bord existe-t-il toujours un an après sa mise en place ? Dans quelle mesure considère-t-il sa mise en place comme une réussite? Quelles en sont les utilisations effectives et qui sont les utilisateurs de l'outil?

Nous ne sommes en revanche pas parvenus à interroger les autres parties prenantes du projet un an après la mise en place de l'outil. La réussite de cette mise en place à un an est de fait appréciée par le seul dirigeant et cela constitue une limite de l'étude.

Le recueil des données a donc reposé sur une pluralité de sources : coconstruction d'un outil de gestion et interactions avec les principaux protagonistes; observation des pratiques et des relations inter et intraorganisationnelles; sources documentaires ; entretiens réalisés pendant et un an après la mise en place de l'outil. Cette pluralité des sources a permis le recueil d’un matériau relativement riche, diversifié et représentatif de la réalité des pratiques.

\subsection{La description du cas}

Cette entreprise, que nous appellerons Phonepro, a vu le jour en 1999 et fut le premier acteur français de vente à distance de matériel de téléphonie pour les entreprises. En 2010, les deux dirigeants-fondateurs, actionnaires majoritaires encore aujourd'hui, nomment le présidentdirecteur général actuel (que nous nommons « dirigeant» dans cet article). La PME comptait en 2012, au moment de l'étude, 54 salariés. L’entreprise, dont la structure et le découpage s'apparentent à un modèle hiérarchico fonctionnel, est organisée en plusieurs services (financier, achat, marketing, commercial, logistique). En plus du dirigeant, l'entreprise compte un responsable pour chaque service. Précisons que le service commercial dispose de deux responsables, l'un d'entre eux étant spécialisé dans la gestion des grands comptes. Hormis la force de vente, qui représente la plus grande part des salariés de l'entreprise, Phonepro est constitué d'un certain nombre d'acteurs clés, aux compétences spécifiques et rattachés aux différents services. On peut notamment citer le chef produit, la chargée de catalogue, l'ingénieur informatique, la responsable des approvisionnements, les deux comptables et le directeur financier (qui a rejoint Phonepro en 2008 et qui a été nommé en 2010 directeur 
administratif et financier en charge de la finance et de la supervision des domaines administratifs hors achats stratégiques et informatiques). Enfin, des employés aux tâches variées sont également présents dans les divers services de l'entreprise.

L'entreprise achète auprès de plusieurs fournisseurs des équipements de téléphonie. Plus précisément, dix fournisseurs représentent environ $80 \%$ du montant total des achats. Toutes les plus grandes marques de téléphonie sont représentées dans l'offre de Phonepro. L’entreprise compte par ailleurs quatre partenaires logistiques majeurs.

Avec un stock disponible sur site de plus de 2000 références produits et accessoires, plus de $96 \%$ des commandes sont expédiées le jour même. Le portefeuille produits s'organise en "familles produits » (casque, talkie-walkie, téléphone filaire, standard téléphonique, solutions collaboratives, etc.).

Les clients sont des professionnels et dans une moindre mesure des particuliers. La communication de l'entreprise repose sur un outil majeur : le catalogue (catalogue clients et catalogue prospects).

Au moment de l'étude, Phonepro faisait face à cinq concurrents principaux et à une multitude de " petits » concurrents. Cette concurrence se caractérise par une stratégie-prix très agressive, ce qui contraint Phonepro à aligner ses prix sur ceux des concurrents et entraîne une forte dégradation de ses marges.

Le dirigeant indique que les principaux facteurs clés de succès de Phonepro sont :

- une communication active, via le site Internet de l'entreprise et les catalogues «clients » et «prospects";

- des délais de livraison rapides;

- une fréquence élevée de lancement de nouveaux produits ;

- une formation continue des salariés ;

- des conseils et SAV gratuits et réactifs ;

- des prix compétitifs.

Avant d'amorcer l'analyse des résultats, précisons que bien que l'un des coauteurs ait participé au processus de conception du tableau de bord achat, l'objet de cet article n'est pas de décrire ce processus, mais de proposer une description de cet outil afin de montrer qu'un outil de contrôle relativement élaboré peut s'avérer adapté aux besoins d'une PME, et d'identifier les caractéristiques organisationnelles, environnementales et comportementales permettant de comprendre la réussite perçue par le dirigeant de cette mise en place.

\section{RÉSULTATS}

Le système de contrôle de Phonepro compte désormais un tableau de bord financier historique mis en place plusieurs années auparavant par le directeur financier et mis à jour par le personnel du service comptable et financier, et cinq tableaux de bord « satellites » : un tableau de bord 
sur les campagnes catalogue, un tableau de bord « Web », un tableau de bord commercial, un tableau de bord logistique et un nouveau tableau de bord achat, objet de notre étude.

Nous présentons dans un premier temps les caractéristiques de ce tableau de bord achat en observant notamment comment il s'articule avec les autres outils de contrôle existants. Nous exposons ensuite la combinaison de caractéristiques organisationnelles, environnementales et individuelles qui explique cette mise en place. Nous proposons enfin des pistes permettant de comprendre pourquoi la mise en place de cet outil est perçue comme une réussite par le dirigeant de Phonepro.

\subsection{Le tableau de bord " achat " : caractéristiques, utilisations et articulation avec les autres outils}

Après avoir proposé une description « opératoire » de l'outil, nous présentons ses principales utilisations et son articulation avec les autres outils de gestion.

\subsubsection{ANALYSE OPÉRATOIRE DU TABLEAU DE BORD ACHAT}

Plusieurs objectifs ont été initialement assignés au tableau de bord achat par la direction et la responsable achat. Il devait tout d'abord permettre le suivi du service ainsi que son pilotage. Il devait ensuite garantir une meilleure gestion des stocks dans le but d'améliorer la trésorerie. Il devait enfin accompagner la mise en place et le suivi d'une démarche globale de gestion de la chaîne logistique et permettre en amont de suivre les fournisseurs et en aval d'améliorer la qualité de service délivrée aux clients. Plus précisément, le dirigeant raconte dans quelle mesure la mise en place d'un outil de contrôle de gestion tel que le tableau de bord s'est avérée nécessaire pour le service achat de son entreprise : "Les objectifs fixés à la responsable achat étaient de mieux appréhender la qualité de service des fournisseurs, ce qu'on ne mesurait pas encore, c'était assez empirique. Mais à un moment donné, pour améliorer les choses, il faut des données. Par exemple, si on souhaite mettre en place des pénalités de retard sur un fournisseur, il faut des données chiffrées. Si on n'a pas d'exemples concrets, on ne fait pas bouger les choses. »

L'objectif était donc de construire un tableau de bord achat constituant un véritable outil d'amélioration continue pour le service auquel il est rattaché et pour l'entreprise dans son ensemble.

Le tableau de bord achat mis en place est ainsi composé de plusieurs « sous-tableaux de bord» :

- un sous-tableau de bord « gestion des achats » : nombre de commandes « achats ", répartition des commandes sur l'année, montant global des achats, répartition du montant des achats sur l'année, délai moyen de livraison aux clients, taux de rupture ;

- un sous-tableau de bord "gestion des stocks » : valeur moyenne du stock, délai moyen de rotation des stocks, taux de rebuts, produits à faible rotation ; 
- un sous-tableau de bord "gestion des références » : nombre de références actives, nombre de nouvelles références, nombre de références obsolètes, nombre de références reconditionnées ;

- un sous-tableau de bord "gestion des fournisseurs » : nombre de commandes par fournisseur, montant des achats par fournisseur, nombre de commandes incomplètes par fournisseur, délai de livraison par fournisseur.

Ces différentes informations sont présentées sous forme de tableaux chiffrés et de graphiques (une présentation du tableau de bord achat dans sa forme chiffrée est proposée en annexe 1).

La réactivité, la diversité de contenu, la nature des objets contrôlés et le degré de décentralisation de l'outil montrent que le tableau de bord achat de Phonepro possède des attributs communs aux tableaux de bord stratégiques et aux tableaux de bord de gestion et peut ainsi recevoir, au sens de Germain (2005, p. 139) «le qualificatif d'outil de pilotage » :

- la réactivité : la «fréquence d'édition » du tableau de bord achat mis en place chez Phonepro est mensuelle. Plus précisément, les données sont actualisées à minima chaque fin de mois, c'est-à-dire qu'elles peuvent être mises à jour à fréquence plus rapide en fonction des besoins. L'actualisation et l'utilisation du tableau de bord se font ainsi au gré des besoins et nécessités. Ceci est notamment permis grâce à Myreport, logiciel de business intelligence, qui assure un fort degré de réactivité de l'outil et par là même de l'entreprise. Le « délai de production ", c'est-à-dire le temps nécessaire à l'actualisation des données, est en effet très bref. Ce critère est apparu comme un facteur clé de succès aux yeux de tous les acteurs impliqués dans le processus ;

- le contenu : des indicateurs de natures très diverses sont présents dans le tableau de bord achat. La valeur du stock ou le montant global des achats témoignent de la présence de données financières. Mais les données non financières telles que les délais de livraison ou le taux de rupture sont majoritaires ;

- les objets contrôlés : l'intérêt majeur du tableau de bord achat réside dans l'existence d'indicateurs non financiers étroitement corrélés aux facteurs clés de succès de l'entreprise et à sa stratégie concurrentielle. L'indicateur « nombre de nouvelles références » mesure par exemple le degré d'innovation dans l'offre de produits proposée aux clients et correspond au positionnement stratégique de l'entreprise : avoir une offre sans cesse innovante et surprenante (une stratégie de pionnier). Les indicateurs « taux de rupture » et « délai de livraison aux clients » sont des indicateurs tournés vers les clients puisqu'ils conditionnent leur niveau de satisfaction. Enfin, l'existence d'un sous-tableau de bord fournisseur représente le degré d'ouverture du tableau de bord achat vers l'extérieur et permet de remplir l'objectif d'une démarche de gestion de la chaîne logistique. En effet, suivre le montant des achats par fournisseur et son évolution permet de mettre en concurrence les fournisseurs. C'est un outil de négociation qui permet de garantir des prix toujours plus bas aux clients et une meilleure qualité de service ;

- le degré de décentralisation : le tableau de bord achat est destiné à la direction ainsi qu’aux différents niveaux fonctionnels et hiérarchiques. En effet, un comité a été créé peu après l'implémentation du tableau de bord achat. Le but étant, selon le dirigeant: « de se retrouver ensemble mensuellement autour de l'outil pour discuter des données ». Plus précisément, le chef de produit, la responsable achat et la personne en charge des approvisionnements, le 
responsable financier, la personne chargée de la partie catalogue et le responsable marketing participent à ces réunions autour de problématiques telles que, par exemple, la gestion des produits à faible rotation. On observe ainsi comment cet outil a su s'affranchir des barrières tant hiérarchiques que fonctionnelles pour profiter au plus grand nombre d'acteurs liés les uns aux autres par des problématiques communes.

\subsubsection{LES PRINCIPALES UTILISATIONS DU TABLEAU DE BORD ACHAT}

L'implémentation du tableau de bord achat résulte de multiples attentes, mais face aux objectifs annoncés, comment l'outil est-il finalement utilisé et quels sont les acteurs concernés ? Pour répondre à cette question, nous avons interrogé le dirigeant de Phonepro un an après la mise en place de l'outil. Au-delà de son utilisation pour la gestion courante du service achat, deux autres utilisations majeures de l'outil apparaissent. Le dirigeant souligne d'une part l'importance de l'utilisation de ce tableau de bord pour la gestion des produits à faible rotation. Il précise que l'implémentation de ce nouvel instrument " a débouché sur une réunion approvisionnement-marketing-finance mensuelle pour voir comment sortir ces produits du stock soit en décidant de les dévaloriser et de les vendre, soit en décidant de s'en débarrasser, mais en tout cas, nettoyer le stock physiquement ». Pour le dirigeant de Phonepro, le tableau de bord achat a ainsi entraîné sur ce point «une plus grande cohésion entre les services et a permis aux acteurs de trouver des solutions ensemble».

Il souligne d'autre part que l'outil est utilisé pour faciliter la relation avec les fournisseurs : "Le tableau de bord est aussi utilisé avec nos fournisseurs, il est nécessaire de leur transmettre les données parce que souvent il y a des problèmes avec certains d'entre eux, on le sait au niveau empirique, mais pas sur les données et sans données concrètes, il ne se passe rien. »

Cette utilisation de l'outil dans le cadre de la relation avec les fournisseurs joue donc un double rôle. Celui de négociation sur l'amélioration de la qualité de service et la réduction des prix, et celui de justification-légitimation d'éventuelles pénalités telles que des pénalités de retard en cas de non-respect des délais de livraison ou des pénalités relatives à l'incomplétude des commandes livrées.

Un an après sa mise en place, le dirigeant estime que le rôle du tableau de bord achat est très important, bien évidemment pour le service achat, mais surtout pour le pilotage de la performance globale de l'entreprise. Les missions qui lui sont attribuées et les nombreux acteurs qu'il sollicite, tant internes qu'externes, lui confèrent une place singulière au sein du système de contrôle de Phonepro.

\subsubsection{LARTICULATION DU TABLEAU DE BORD ACHAT AVEC LES AUTRES OUTILS DE GESTION}

Si le tableau de bord achat semble remplir plusieurs fonctions, nos observations ont montré que celles-ci s'exerçaient en complément de celles remplies par d'autres outils. La mise en place d'une démarche de gestion de la chaîne logistique a fait apparaître la nécessité de sélectionner d'autres indicateurs que ceux contenus dans le tableau de bord financier. Le tableau de bord financier historique et le tableau de bord achat nouvellement mis en place apparaissent comme très complémentaires tant leur contenu et leurs buts diffèrent. Le premier est composé d'un vaste ensemble d'indicateurs financiers, dont le but est de révéler la 
santé financière de l'entreprise sous divers angles, et de fournir les informations attendues par les actionnaires (rôle de légitimation du dirigeant), et celles nécessaires aux documents comptables officiels. Le second contient un panel d'indicateurs beaucoup plus variés. Son rôle relève davantage du pilotage de l'entreprise et de la performance globale que du contrôle des résultats financiers.

Le tableau de bord achat de Phonepro cohabite avec d'autres outils encore : un tableau de bord propre aux campagnes catalogue, un tableau de bord «Web», un tableau de bord commercial, un tableau de bord logistique ainsi que Myreport, outil de business intelligence qui permet d'avoir accès à un très grand nombre de données concernant l'activité et le fonctionnement de l'entreprise. La très grande majorité d'entre eux a été mise en place par le dirigeant qui nous explique: "mes prédécesseurs avaient peu d'outils, car ils avaient créé l'entreprise et ils géraient beaucoup au flair. Quand je suis arrivé, il y en avait peu, je fonctionnais un peu à l'aveugle. Nous en avons donc mis quelques-uns en place, mais pas trop. J'en avais besoin pour prendre les décisions parce que je n'avais pas assez de visibilité ». Il considère ainsi que "désormais Phonepro est bien outillée ». Au vu de la littérature sur la PME, le système de contrôle de cette entreprise semble relativement élaboré, mais aux yeux de son dirigeant, "Phonepro n'est pas une entreprise où il y a énormément de tableaux de bord, il y a l'essentiel ». Il considère les tableaux de bord commercial, logistique et marketing, comme un complément qualitatif indispensable aux indicateurs de performance financière présents au sein du tableau de bord financier. Néanmoins, ces tableaux de bord opérationnels, composés d'indicateurs spécifiques à chaque service, apparaissent comme des instruments de pilotage locaux, peu adaptés à la démarche de gestion de la chaîne logistique dans laquelle le dirigeant et la responsable achat souhaitaient s'inscrire. Le tableau de bord achat, en intégrant des indicateurs étroitement corrélés aux facteurs clés de succès de l'entreprise et à sa stratégie concurrentielle, facilite le pilotage global de la performance de l'entreprise et apparaît donc comme complémentaire aux autres instruments en place.

Le dirigeant de Phonepro s'est ainsi doté d'un véritable outil managérial relativement élaboré au sens de Germain (2005), proche de ceux susceptibles d'être observés dans des organisations de taille plus importante. Sous l'influence de divers facteurs, Phonepro s'est donc inscrite dans une démarche d'implémentation d'outils de contrôle. Ces outils pour certains loin d'être simples et rudimentaires, répondent à des besoins managériaux précis et s'adaptent au contexte unique et singulier de l'entreprise. Et c'est précisément parce que l'entreprise et ses acteurs se les sont appropriés qu'ils impulsent des actions concrètes, répondent aux besoins et objectifs initiaux et perdurent dans le temps. Nous décrivons dans la partie suivante les caractéristiques organisationnelles, environnementales et comportementales permettant de comprendre la mise en place de cet outil dans cette PME d'une cinquantaine de salariés.

\subsection{Une combinaison de caractéristiques permettant de comprendre la mise en place du tableau de bord achat}

Puisque Phonepro compte 54 salariés, on pouvait s'attendre à ce que son système de contrôle soit le reflet des pratiques énoncées par une majorité d'auteurs (Davila, 2005 ; Zawadzki, 2011), c'est-à-dire simple et informel. Plusieurs éléments viennent nuancer, chez Phonepro, 
l'influence que peut avoir la taille sur le système de contrôle et conduisent à une situation à la fois surprenante (au regard de la littérature) et atypique.

\subsubsection{UN DIRIGEANT DE FORMATION GESTIONNAIRE ET DISPOSANT D'UNE EXPÉRIENCE EN GRANDE ENTREPRISE}

Le dirigeant de Phonepro a été à l'initiative, avec la responsable du service achat, de la mise en place du tableau de bord achat.

Ce dirigeant dispose d'une formation universitaire en gestion. Son parcours l'a, de son propre aveu, imprégné de la « doxa gestionnaire » et l'a conduit à mettre en place dans son entreprise les outils de gestion qui lui ont été enseignés pendant ses années passées à l'Université. Cette observation peut être interprétée comme le fruit de pratiques isomorphes, voire mimétiques, liées à la formation des dirigeants poussant à l'adoption de pratiques de gestion identiques inculquées sur les bancs des universités ou des écoles de commerce.

Par ailleurs, son expérience professionnelle préalable a aussi influencé le choix de l'outil puisqu'il a auparavant travaillé dans une entreprise de près de 1000 salariés et dans une société cotée en bourse de 10000 employés. Les outils de gestion rencontrés dans les grandes structures lui sont donc familiers. Le dirigeant de Phonepro relate en effet : "Le tableau de bord achat, je l'ai demandé à la responsable achat, car dans mes anciennes boîtes, on avait mis en place des tableaux de bord fournisseurs et ça nous permettait de faire bouger nos fournisseurs sur la qualité de leur service». Ainsi, ces expériences ont influencé son choix de mettre en place chez Phonepro un tableau de bord achat relativement élaboré, et explique la mise en place de ce tableau de bord achat comme un transfert de pratiques.

\subsubsection{LES PERSPECTIVES ET OBJECTIFS DE CROISSANCE DE LA FIRME}

Phonepro n'est pas aujourd'hui dans une logique d'indépendance patrimoniale, mais davantage dans une logique de croissance. Ses perspectives sont claires : continuer à croître et augmenter ses parts de marché par-delà les frontières. Or, être dans une volonté et démarche de croissance induit une complexification de l'organisation avec de plus en plus d'employés, de services et de niveaux hiérarchiques à gérer. Le suivi et le contrôle de la bonne marche de l'entreprise, la communication, la motivation, le pilotage de l'activité et des stratégies dans une telle optique ne peuvent plus être assurés de manière trop informelle. En ce sens, la direction de Phonepro a exprimé un besoin de formalisation de son système de contrôle justifiant, entre autres, la mise en place du tableau de bord achat.

\subsubsection{LA PRÉSENCE D'UN PROGICIEL DE GESTION INTÉGRÉ (PGI) ET D'UN INGÉNIEUR INFORMATIQUE}

Phonepro dispose d'un PGI récemment mis en place et paramétré dans le but de doter l'organisation et chacun de ses membres d'un grand nombre d'informations dans un très bref délai. Ce PGI, qui enregistre, stocke et traite les informations relatives à l'entreprise, a permis la mise en place du tableau de bord achat. Il constitue une source d'informations relativement riche qui alimente le tableau de bord. Disposer d'un PGI permet ainsi, à tout moment, de dresser, dans un délai très court, un historique d'un ou plusieurs indicateurs jugés importants. Disposer d'un PGI permet aussi l'automatisation des calculs des indica- 
teurs constituant le tableau de bord. Ceci engendre un gain de temps et un gain de fiabilité de l'information et contribue à maintenir à jour cet outil de contrôle et de prise de décision, conditions importantes de sa durabilité et de sa légitimité.

L'observation participante a par ailleurs révélé l'importance de la présence d'un ingénieur informatique qui a facilité l'implémentation et l'automatisation des indicateurs retenus. Les solutions techniques qu'il a su proposer ont constitué un des éléments clés du succès de la mise en place du tableau de bord achat.

\subsubsection{LA MISE EN PLACE D'UNE DÉMARCHE DE GESTION DE LA CHAÎNE LOGISTIQUE}

Peu avant l'observation participante et la mise en place du tableau de bord achat, la direction de Phonepro a exprimé le besoin de mettre en place une démarche de gestion de la chaîne logistique. Cette démarche, perçue comme un facteur décisif d'avantage concurrentiel, a justifié la mise en place du tableau de bord achat, car elle a fait apparaître la nécessité de sélectionner d'autres indicateurs que ceux contenus dans le tableau de bord financier. Ainsi, lorsque le dirigeant de Phonepro répond à la question des changements imputables au nouvel outil, il explique : "On a par exemple pu rencontrer des fournisseurs et réaliser des négociations à partir des données du tableau de bord. On s'est servi de ce tableau de bord pour montrer quels étaient les problèmes et demander des améliorations en termes de prix, mais surtout en termes de qualité de services ». Cette démarche s'inscrit pleinement dans une problématique d'alignement stratégique telle que définie par Kaplan et Norton (2007) et visant à assurer un suivi des relations fournisseurs dans une démarche de gestion de la chaîne logistique (Alcouffe, Boitier, Rivière et Villesèque-Dubus, 2013). Les tableaux de bord fournisseurs décrits par Kaplan et Norton s'inscrivent en effet dans une démarche interfonctionnelle, mais aussi interorganisationnelle de l'entreprise, et semblent de ce point de vue correspondre à la vision du dirigeant de Phonepro, lequel accorde une place particulièrement importante au suivi des relations fournisseurs.

\subsubsection{UNE STRUCTURE ORGANISATIONNELLE HIÉRARCHISÉE AVEC DES SALARIÉS DISPOSANT D'UNE FORTE TECHNICITÉ}

La structure de Phonepro est hiérarchique par opposition à un organigramme dit "écrasé ", ou structure simple qui selon Mintzberg (1982) caractérise la PME. Un responsable est en place à la tête de chaque service. L'entreprise compte ainsi un responsable achat, un responsable informatique, un responsable logistique, un responsable marketing, un responsable financier, et deux responsables commerciaux. Par ailleurs, on observe dans les services financiers, achat et marketing, la présence de sous-responsables attachés à des fonctions différentes.

De plus, les salariés ne sont pas polyvalents. Ils sont spécialisés et disposent d'une forte technicité et souvent d'un savoir-faire unique. Chacun représente ainsi un maillon précieux d'une chaîne fragile et complexe. La forte imbrication des services les uns avec les autres et la forte spécialisation des individus les rendent indispensables à la bonne marche de l'entreprise. 
Cette structure organisationnelle explique pour partie la configuration particulière du système de contrôle chez Phonepro. Le fait que les différents services soient séparés, mais interdépendants implique une gestion rigoureuse et la mise en place d'outils formalisés. Chaque responsable de service doit rendre des comptes au dirigeant et informer les responsables des autres services auxquels il est relié. La responsable du service achat par exemple est en relation avec les responsables de tous les services. L'impact important des achats et des stocks sur la trésorerie oblige la responsable achat et le responsable financier à se rencontrer régulièrement et à prendre des décisions à partir d'indicateurs majeurs présents dans le tableau de bord achat. De même, des indicateurs tels que le nombre de références obsolètes ou le délai de rotation des stocks obligent la responsable achat et le responsable commercial à travailler ensemble et pourront conduire à des prises de décisions telles que « casser les prix sur le produit $\mathrm{X}$ » ou bien « un produit $\mathrm{Y}$ acheté, un produit $\mathrm{X}$ offert »...

Au final, la structure organisationnelle de Phonepro composée de services fonctionnels séparés et interdépendants reflète un contexte bien particulier de PME et explique pour partie la mise en place de ce tableau de bord achat. Cet outil ne rend pas seulement compte de l'activité du service achat, il facilite aussi les relations entre ce service et les autres services.

\subsubsection{UN ENVIRONNEMENT PERÇU COMME HOSTILE ET VERSATILE}

La conjoncture économique étant difficile, la place de leader de Phonepro est chaque jour menacée par la présence sur le marché de cinq concurrents majeurs, mettant en œuvre des stratégies agressives en termes de prix.

Pour faire face à cette situation, Phonepro a mis en place un système de contrôle permettant de surveiller l'activité et d'assurer la performance de l'entreprise en déjouant les menaces et en mettant à jour les opportunités de son environnement. Par exemple, pour résoudre un problème de dégradation de la marge sur certains produits occasionné par l'alignement sur les prix des concurrents, Phonepro a renforcé les négociations avec les fournisseurs sur le prix d'achat. Le tableau de bord achat permet de rendre compte de ce nouvel impératif. Cette vive concurrence a également conduit Phonepro à suivre d'autres facteurs clés de succès qui, selon le dirigeant, la différencient de ses concurrents comme le délai de livraison ou le taux de rupture. L’environnement jugé hostile a poussé l'entreprise à prendre des décisions stratégiques qui ont impacté les outils de contrôle de gestion en général et justifié la nécessité de mettre en place un tableau de bord achat.

\subsection{Un outil perçu comme une réussite par le dirigeant de Phonepro}

Quelques années avant la mise en place de cet outil, une première tentative de mise en œuvre d'un tableau de bord achat avait été lancée sans succès chez Phonepro. Il est ressorti de l'analyse de cet échec que le tableau de bord mis en place contenait tout d'abord un trop grand nombre d'indicateurs limitant sa lisibilité et donc son efficacité. La responsable achat indique à ce sujet que "les voyants majeurs étaient noyés parmi un vaste ensemble d'indicateurs secondaires ». Ces indicateurs étaient par ailleurs manuels. L'ingénieur informatique n’avait pas été sollicité et aucune automatisation des calculs n’avait été prévue. Cet outil n'avait enfin pas été le fruit d'une construction et d'une volonté collective. Quelques parties 
prenantes n'avaient pas été impliquées à sa conception et y étaient pour certaines opposées. In fine, ce tableau de bord jugé par certains illisible, par d'autres très lourd à actualiser, avait été rapidement abandonné.

Quelques années après, le tableau de bord achat observé dans le cadre de cette recherche a été mis en place à l'initiative du dirigeant et de la responsable achat. Ces deux acteurs ont dès le départ cru en l'utilité de cet outil et ont communiqué leur volonté de le mettre en place à l'ensemble des parties prenantes. Il a été le fruit d'une coconstruction entre un chercheur externe et plusieurs salariés de l'entreprise. Le chercheur a en effet été l'interface active entre la direction, l'ingénieur informatique maîtrisant l'accès aux données et les rendant exploitables sous forme d'informations, la responsable achat et les employés du service achat au fait des principaux enjeux et difficultés du service et du réseau de la chaîne logistique. Ensemble, les différentes parties prenantes ont œuvré à la sélection d'un nombre significatif, mais limité d'indicateurs de gestion de l'activité du service. Forte de l'expérience de l'échec passé, l'entreprise s'est lancée dans la construction de ce nouvel outil sans reproduire les mêmes erreurs. Ce tableau de bord achat relativement élaboré a été adapté aux caractéristiques de l'entreprise, de son environnement, et au profil de son dirigeant et des différentes parties prenantes. Le dirigeant perçoit la mise en place de cet outil comme une réussite du fait qu'il ait impulsé des actions concrètes sur des problématiques telles que la négociation fournisseurs, la gestion des prestataires logistiques ou la gestion des stocks.

Il explique au sujet de la relation avec les fournisseurs que "lorsque les commerciaux discutaient avec les fournisseurs de problèmes de livraison sans données concrètes, il ne se passait rien, mais le jour où les commerciaux ont pu s'appuyer sur le tableau de bord chiffré, ça a permis des améliorations». Il ajoute que "le fait de montrer et quantifier les problèmes tels que les retards de livraison ou le nombre de commandes incomplètes avec les pénalités associées, et de dresser des comparaisons avec les résultats de certains concurrents plus efficaces, a effectivement fait bouger les choses. Les résultats de nos fournisseurs sont dans l'ensemble maintenant bien meilleurs ». Le sous-tableau de bord " gestion des fournisseurs » a ainsi répondu à l'objectif stratégique de mettre en œuvre une démarche de gestion de la chaîne logistique. En effet, les indicateurs élaborés chaque fin de mois dans ce tableau permettent d'établir le lien avec les parties prenantes externes afin d'optimiser l'ensemble de la chaîne de valeur. Aux yeux du dirigeant, le fait que les négociations fournisseurs, sur la base du tableau de bord achat, aient débouché sur une amélioration sensible des délais de livraison et du nombre de commandes incomplètes, impacte in fine la satisfaction des clients. Il considère ainsi que l'objectif d'inscrire Phonepro dans une démarche de gestion de la chaîne logistique est au moins en partie respecté et qu'en ce sens, "la mise en place du tableau de bord achat est un pari réussi ».

Ce nouvel outil a par ailleurs permis la mise en place d'un suivi des prestataires logistiques (via le sous-tableau de bord fournisseur) afin d'améliorer là encore les délais de livraison. Une discussion a notamment débuté très vite après la mise en place de l'outil, sur la base des chiffres fournis par le tableau de bord, avec un des quatre prestataires logistiques de Phonepro afin qu'il améliore ses délais. La responsable achat affirmait ainsi que si le prestataire n'avait pas amélioré ses performances, une interruption du partenariat aurait été envisagée. 
En ce qui concerne la gestion des stocks, le dirigeant affirme enfin que : "c'est le même cas que pour les fournisseurs, quand on dit au service marketing par exemple qu'il y a des produits qui ne se vendent pas, il ne se passe rien concrètement. Alors que lorsqu'on a commencé chaque mois à montrer la liste des références, avec leur délai de rotation et les montants associés, on a tout de suite mis en place des actions sur les dix plus mauvaises références... Il s'agissait plus précisément de décisions de mises au rebut ou de diverses actions commerciales, comme réaliser des rabais, des ventes en lots, ou encore des opérations de communication pour mettre en avant les produits en difficulté».

Les informations auxquelles il fait référence sont celles du tableau présenté ci-dessous :

TABLEAU 1. GeStion DES PRODUits À FAIBLE ROTATION

\begin{tabular}{llllll}
\hline Classe & Nom & $\begin{array}{l}\text { Délai de rotation } \\
\text { du stock en } \\
\text { jours (ordre } \\
\text { décroissant) }\end{array}$ & $\begin{array}{l}\text { Valeur du } \\
\text { stock }\end{array}$ & $\begin{array}{l}\text { \% de la valeur } \\
\text { du stock total }\end{array}$ & $\begin{array}{l}\text { \% cumulé de la } \\
\text { valeur du stock } \\
\text { total }\end{array}$ \\
\hline A & Réf 1 & & & \\
& Réf 2 & & \\
& $\ldots$ \\
& Réf 10 & & \\
\hline B & Ref 11 & & \\
& Réf 12 & & \\
& $\ldots$ & & \\
& Réf 20 & & \\
\hline C & Ref 21 & & \\
& Réf 22 & & \\
& $\ldots$ & & \\
\hline
\end{tabular}

Chaque fin de mois, les références, dont la valeur en stock est importante (le tableau ne recense pas les références, dont la valeur du stock est faible) et, dont les délais de rotation sont anormalement importants, sont identifiées et classées en trois sous-groupes par ordre décroissant selon leur délai de rotation (la première référence a une valeur en stock importante et le délai de rotation le plus long) :

- A : les dix références, dont les délais de rotation sont les plus importants (les produits qui ne se vendent pas);

- B : les dix produits suivants (ce ne sont pas les pires, mais ils posent problème...);

- C : les produits suivants jusqu'à atteindre une valeur cumulée de $80 \%$ de la valeur du stock total.

Ce tableau permet chaque fin de mois à la responsable achat, au directeur financier et au responsable commercial, d'identifier les produits qui posent problème et sur lesquels il faut agir immédiatement : ceux qui ont une valeur élevée et une faible rotation. Le mois suivant, les produits de la classe A retrouvent le plus souvent des délais de rotation satisfaisants. L'attention se porte 
alors sur les nouvelles références de classe A (qui sont souvent les références de classe B du mois précédent).

Au final, selon le dirigeant, "le tableau de bord achat correspondait à un réel besoin pour le service achat dans son ensemble et pour les personnes chargées de la gestion des stocks et des fournisseurs en particulier». Il affirme que "l'accueil de l'outil a été positif, il est perçu comme utile par les acteurs concernés ». La construction de ce nouveau tableau de bord a été à ses yeux le fruit d'un apprentissage collectif et, tel un cercle vertueux, a contribué à son tour à l'apprentissage collectif (Argyris, 2000).

\section{DISCUSSION ET CONCLUSION}

Les recherches empiriques réalisées sur le thème du système de contrôle du dirigeant de PME aboutissent à des conclusions qui pourraient sembler contradictoires. Certaines montrent que les dirigeants des entreprises les plus petites disposent de systèmes de contrôle simples et peu formalisés (Davila, 2005 ; Zawadzki, 2011). Mais d'autres soulignent que certains dirigeants de PME disposent de systèmes de contrôle relativement élaborés, parfois proches de ceux, dont disposent les grandes entreprises (Lavigne, 2002; Affès et Chabchoub, 2007).

Nous pensons que ces deux pans de la littérature sont complémentaires et non contradictoires. Chapellier et Dupuy (2013) et Oriot et Bergeron (2015) montrent que, dans certaines PME, les systèmes de contrôle sont simples et peu formalisés non parce que ces données sont jugées inutiles ou peu pertinentes par les dirigeants, mais pour être adaptées au contexte, aux besoins et aux compétences. Chapellier et Dupuy (2013, p. 145) expliquent que «la simplicité apparente de la forme n'occulte pas la complexité de sens. Elle en conditionne au contraire l'émergence » et qu' "un système de contrôle très développé et formalisé, qui véhiculerait implicitement la référence à une rationalité plus ou moins substantielle, ne ferait finalement pas sens pour ce type de dirigeants". Ceux-ci disposent de tableaux de bord plutôt « rudimentaires " adoptant souvent la forme d'un compte de résultat, ou d'un tableau de bord de suivi budgétaire financier et comptable.

Notre étude montre que d'autres dirigeants, comme celui de Phonepro, disposent en revanche de systèmes de contrôle plus élaborés et plus formalisés, assez proches de ceux observés dans les grandes entreprises, parce que les caractéristiques organisationnelles et environnementales de leur PME et leur profil (notamment en termes de formation et d'expérience), le rendent possible, voire nécessaire. Ceux-là peuvent disposer de tableaux de bord plus formalisés, réactifs, décentralisés, orientés vers les parties prenantes externes, et en lien avec la stratégie (Germain, 2005).

Ce cas unique, bien que riche d'enseignements, ne nous permet pas de généraliser nos résultats, mais soutient l'idée que les données de contrôle prennent du sens aux yeux des dirigeants de PME sous condition d'adaptation. Nous rejoignons ainsi Chapellier et Dupuy (2013, p. 145) qui affirment que "les dirigeants de PME ont besoin des données de contrôle de gestion adaptées pour assurer leur intelligence comptable, c'est-à-dire leur capacité à distinguer les problèmes à partir de ces données ». Cette adaptation relève d'une mise en adéquation avec des facteurs de contingence organisationnels, environnementaux, et individuels. Les caractéristiques de certaines PME et le profil de leur dirigeant impliquent la mise en place d'outils de contrôle simples ("sans être simplistes ", Oriot et Bergeron, 2015, p. 180) et peu formalisés. Les caractéristiques d'autres PME, telles que Phonepro, et le profil de leur dirigeant, peuvent conduire à la mise en place d'outils de 
contrôle relativement élaborés, réactifs, décentralisés, composés d'indicateurs financiers et non financiers en lien avec la stratégie (Oriot et Bergeron, 2012).

Notre étude montre par ailleurs la grande capacité d'adaptation des tableaux de bord et confirme les résultats de Germain (2001) qui explique que cet outil présente des attributs le rendant parfaitement approprié au contexte PME. Pour l'auteur, "le tableau de bord fournit une certaine représentation de l'entreprise, plus concrète et plus complète que celle induite par les outils traditionnels de contrôle de gestion... Échappant à tout cadre normatif, l'outil peut facilement être conçu selon des schémas ad hoc et être personnalisé de manière à répondre très précisément aux besoins de l'utilisateur "(Germain, 2001, p. 5). Mais cela suppose que ses concepteurs s'inscrivent dans une démarche d'adaptation de l'outil en fonction des besoins, des ressources et de l'environnement de la PME. La diversité des situations observées dans le monde de la PME nous conduit à penser, comme Oriot et Misiaszek (2012, p. 39) que «c'est aux systèmes de management de la performance de s'adapter aux réalités des différentes PME et non à ces dernières de passer sous les fourches caudines de modèles prédéfinis quels qu'ils soient ». Notre étude soutient ainsi l'idée que la diversité des contextes et des acteurs invite les experts internes (notamment les contrôleurs de gestion) ou externes (notamment les experts-comptables et consultants), à tenir compte des spécificités organisationnelles, environnementales et individuelles quand ils mettent en place des outils de gestion en PME.

La prudence doit cependant présider à l'interprétation et à l'appréciation de nos conclusions compte tenu des limites de l'étude. Il s'agit en premier lieu d'une étude portant sur une seule PME. Il conviendrait de réitérer ce travail afin d'observer d'autres PME évoluant dans d'autres contextes organisationnels et environnementaux.

Ensuite, la perception de la réussite de la mise en place de ce nouvel outil repose sur un seul et unique regard, celui du dirigeant, puisque les chercheurs n'ont pas eu la possibilité d'interviewer, un an après sa mise en place, les autres parties prenantes. Il aurait été très intéressant de recueillir d'autres points de vue pour déterminer dans quelle mesure le tableau de bord achat est perçu comme une « réussite " par l'ensemble des acteurs concernés par l'outil. Ce point représente une limite de notre étude.

De même, seul le tableau de bord achat nouvellement mis en place a été étudié dans cette recherche. Dans la mesure où la problématique concerne les outils de contrôle au sens large, il eut été intéressant de tous les étudier dans le contexte propre à cette PME. Mais la mission du chercheur se situait dans le service achat et l'accès aux données et aux acteurs des autres services n'a pas été suffisant pour qu'une étude de l'ensemble du système de contrôle soit possible. Ce point représente lui aussi une limite de l'étude.

Enfin, nous ne nous sommes pas intéressés à la question du lien entre le système de contrôle et la performance de la PME. Or cette question certes redoutable reste fondamentale.

Ces limites ouvrent des perspectives de recherches intéressantes que nous comptons exploiter à travers la mise en œuvre de nouvelles études portant sur les systèmes de contrôle en PME. Une multiplication d'analyses en profondeur permettrait en effet de mieux comprendre comment, avec un système de contrôle adapté à leurs besoins, au contexte et aux compétences, une fraction significative de dirigeants de PME parvient à des représentations riches et variées leur permettant de piloter efficacement leur organisation. 
ANNeXe 1. TABlEAU DE BORD ACHAT SOUS FORME ChIFFrÉE

\section{GESTION DES ACHATS}

\begin{tabular}{|c|c|c|c|c|c|c|}
\hline & \multicolumn{2}{|c|}{ Janvier } & \multicolumn{2}{|c|}{ Février } & \multicolumn{2}{|c|}{$\ldots$} \\
\hline & $\mathrm{N}$ & $\mathrm{N}-1$ & $\mathrm{~N}$ & $\mathrm{~N}-1$ & $\mathrm{~N}$ & $\mathrm{~N}-1$ \\
\hline $\begin{array}{l}\text { Nombre de } \\
\text { commandes passées } \\
\text { aux fournisseurs }\end{array}$ & & & & & & \\
\hline $\begin{array}{l}\text { Répartition des } \\
\text { commandes sur } \\
\text { l'année (\% par mois) }\end{array}$ & & & & & & \\
\hline Montant achats $(\mathrm{k} €)$ & & & & & & \\
\hline $\begin{array}{l}\text { Répartition montant } \\
\text { achat }(\% \text { par mois })\end{array}$ & & & & & & \\
\hline $\begin{array}{l}\text { Délai moyen de } \\
\text { livraison aux clients } \\
\text { (en jours) }\end{array}$ & & & & & & \\
\hline Taux de rupture $(\%)$ & & & & & & \\
\hline
\end{tabular}

\section{GESTION DES STOCKS}

\begin{tabular}{llllllll}
\cline { 2 - 6 } & \multicolumn{2}{c}{ Janvier } & \multicolumn{2}{c}{ Février } & \multicolumn{2}{c}{$\ldots$} \\
\hline $\mathrm{N}$ & $\mathrm{N}-1$ & $\mathrm{~N}$ & $\mathrm{~N}-1$ & $\mathrm{~N}$ & $\mathrm{~N}-1$ \\
\hline
\end{tabular}

Valeur moyenne du stock $(€)$

Délai moyen de rotation du stock

Taux de rebut (\%)

\section{GESTION DES PRODUITS À FAIBLE ROTATION}

\begin{tabular}{llllll}
\hline Classe & Nom & $\begin{array}{l}\text { Délai de rotation } \\
\text { du stock en jours } \\
\text { (ordre décroissant) }\end{array}$ & $\begin{array}{l}\text { Valeur du } \\
\text { stock }\end{array}$ & $\begin{array}{l}\text { \% de la valeur } \\
\text { du stock total }\end{array}$ & $\begin{array}{l}\text { \% cumulé de la } \\
\text { valeur du stock } \\
\text { total }\end{array}$ \\
\hline A & $\begin{array}{l}\text { Réf } 1 \\
\text { Réf } 2\end{array}$ & & & \\
& $\ldots$ & & & \\
& Réf 10 & & & \\
\end{tabular}




\begin{tabular}{llllll}
\hline Classe & Nom & $\begin{array}{l}\text { Délai de rotation } \\
\text { du stock en jours } \\
\text { (ordre décroissant) }\end{array}$ & $\begin{array}{l}\text { Valeur du } \\
\text { stock }\end{array}$ & $\begin{array}{l}\text { \% de la valeur } \\
\text { du stock total }\end{array}$ & $\begin{array}{l}\text { \% cumulé de la } \\
\text { valeur du stock } \\
\text { total }\end{array}$ \\
\hline B & Ref 11 & & & \\
& Réf 12 & & & \\
& $\ldots$ & & & \\
& Réf 20 & & \\
\hline C & Ref 21 & & \\
& Réf 22 & & \\
& $\ldots$ & & \\
\hline
\end{tabular}

\section{GESTION DES RÉFÉRENCES}

\begin{tabular}{lllllll} 
& \multicolumn{1}{l}{} & Janvier & & Février & & $\ldots$ \\
\cline { 2 - 6 } & $\mathrm{N}$ & $\mathrm{N}-1$ & $\mathrm{~N}$ & $\mathrm{~N}-1$ & $\mathrm{~N}$ & $\mathrm{~N}-1$ \\
\hline Nombre de références actives & & & & & & \\
\hline Nombre de nouvelles références & & & & & & \\
\hline Nombre de références obsolètes & & & & & & \\
\hline $\begin{array}{l}\text { Nombre de références recondi- } \\
\text { tionnées }\end{array}$ & & & & & & \\
\hline
\end{tabular}

\section{GESTION DES FOURNISSEURS}

\section{Nombre de commandes par} fournisseur

\begin{tabular}{|c|c|c|c|c|c|c|}
\hline & \multicolumn{2}{|c|}{ Janvier } & \multicolumn{2}{|c|}{ Février } & \multicolumn{2}{|c|}{$\ldots$} \\
\hline & $\mathrm{N}$ & $\mathrm{N}-1$ & $\mathrm{~N}$ & $\mathrm{~N}-1$ & $\mathrm{~N}$ & $\mathrm{~N}-1$ \\
\hline \multicolumn{7}{|l|}{ Fournisseur 1} \\
\hline \multicolumn{7}{|l|}{ Fournisseur 2} \\
\hline \multicolumn{7}{|l|}{ Fournisseur 3} \\
\hline \\
\hline \multirow{2}{*}{\multicolumn{7}{|c|}{$\begin{array}{l}\text { Montant des achats par } \\
\text { fournisseur }(k €)\end{array}$}} \\
\hline & & & & & & \\
\hline & \multicolumn{2}{|c|}{ Janvier } & \multicolumn{2}{|c|}{ Février } & \multicolumn{2}{|c|}{$\cdots$} \\
\hline & $\mathrm{N}$ & $\mathrm{N}-1$ & $\mathrm{~N}$ & $\mathrm{~N}-1$ & $\mathrm{~N}$ & $\mathrm{~N}-1$ \\
\hline \multicolumn{7}{|l|}{ Fournisseur 1} \\
\hline \multicolumn{7}{|l|}{ Fournisseur 2} \\
\hline \multicolumn{7}{|l|}{ Fournisseur 3} \\
\hline$\cdots$ & & & & & & \\
\hline
\end{tabular}




\section{GESTION DES FOURNISSEURS}

\section{Nombre de commandes}

incomplètes par fournisseur

\begin{tabular}{llllllll}
\hline & \multicolumn{3}{c}{ Janvier } & \multicolumn{2}{c}{ Février } & \multicolumn{2}{c}{.. } \\
\cline { 2 - 7 } & $\mathrm{N}$ & $\mathrm{N}-1$ & $\mathrm{~N}$ & $\mathrm{~N}-1$ & $\mathrm{~N}$ & $\mathrm{~N}-1$ \\
\hline
\end{tabular}

Fournisseur 1

Fournisseur 2

Fournisseur 3

\begin{tabular}{|c|c|c|c|c|c|c|}
\hline \multirow{3}{*}{$\begin{array}{l}\text { Délai de livraison par } \\
\text { fournisseur }\end{array}$} & \multirow{2}{*}{\multicolumn{2}{|c|}{ Janvier }} & \multirow{2}{*}{\multicolumn{2}{|c|}{ Février }} & \multirow{2}{*}{\multicolumn{2}{|c|}{$\ldots$}} \\
\hline & & & & & & \\
\hline & $\mathrm{N}$ & $\mathrm{N}-1$ & $\mathrm{~N}$ & N-1 & $\mathrm{N}$ & $\mathrm{N}-1$ \\
\hline \multicolumn{7}{|l|}{ Fournisseur 1} \\
\hline \multicolumn{7}{|l|}{ Fournisseur 2} \\
\hline \multicolumn{7}{|l|}{ Fournisseur 3} \\
\hline
\end{tabular}




\section{RÉFÉRENCES}

Abdel-Kader, M. et Luther, R. (2008). The impact of firm characteristics on management accounting practices : a UK-based empirical analysis. The British Accounting Review, 40(1), 2-27.

Affès, H. et Снавсноub, A. (2007). Le système d'information comptable : les déterminants de ses caractéristiques et son impact sur la performance financière des PME en Tunisie. Revue des Sciences de Gestion, (224-225), 59-68.

Alcouffe, S., Boitier, M., Rivière, A. et Villesèque-Dubus, F. (2013). Contrôle de gestion interactif: commercial supply chain RH environnement. Paris, Dunod.

ARgYRIS, C. (2000). Savoir pour agir, surmonter les obstacles à l'apprentissage. Paris, Dunod.

Beldi, A. et Cheffi, W. (2005). La comptabilité de gestion : outil d'information ou dispositif de connaissance ? La perception des managers. Actes du $26^{e}$ Congrès de l'Association francophone de comptabilité. Lille, 26-28 mai.

Bergeron, H. (2002). La gestion stratégique et les mesures de la performance non financière des PME. Actes du $6^{e}$ Congrès international francophone sur la PME. Montréal, 30 octobre- $\mathrm{1}^{\mathrm{er}}$ novembre.

Bescos, P.L. et Mendoza, C. (1998). Les besoins d'information des managers sont-ils satisfaits. Revue française de gestion, (121), 117-128.

Bonache, A., Chapellier, P., Ben Hamadi, Z. et Mohammed, A. (2015). Les déterminants de la complexité des systèmes d'information comptables des dirigeants de PME : contingences culturelles et endogénéité. Management International, 19(3), 145-165.

Boulianne, E. (2007). Revisiting fit between AIS design and performance with the analyzer strategictype. International Journal of Accounting Information Systems, 8(1), 1-16.

Chapellier, P. (2003). Les apports d'Internet à la mission de l'expert-comptable dans les petites entreprises. Comptabilité Contrôle Audit, 9(2), 171-187.

Chapellier, P. (2011). Vers un modèle de gestion hybride pour le dirigeant de PME : une étude de la triangulation entre système d'information formel, recours à l'expert et métis du dirigeant (habilitation à diriger des recherches en sciences de gestion). Université de Nice, France.

Chapellier, P. et Ben Hamadi, Z. (2012). Le système de données comptables des dirigeants de PME tunisiennes : complexité et déterminants. Management International, 16(4), 151-165.

Chapellier, P. et Dupuy, Y. (2013). L'hybridation du système de gestion du dirigeant de la PME pérenne. Dans S. Mignon (dir.), Pilotage de la pérennité organisationnelle : normes, représentations et contrôle (p. 135-162). Cormelles-le-Royal, Éditions EMS.

Chapellier, P., Mohammed, A. et Teller, R. (2013). Étude du système d'information comptable des dirigeants de PME syriennes : complexité et contingences. Revue management et avenir, (65), 48-72.

Cheffi, W. et Nekhili, M. (2011). Rôles assignés à la comptabilité de gestion par les managers et changement comptable : question d'adéquation ou simple désillusion ? Comptabilité Contrôle Audit, (1), 67-97.

Chenhall, R.H. (2003). Management control systems design within its organizational context : findings from contingency-based research and directions for the future. Accounting, Organizations and Society, 28(2), 127-168. 
Coker, J.W. et Hayes, R.D. (1992). Lenders' perceptions of income-tax-basis financial reporting by small business. Journal of Small Business Management, 30(3), 66-76.

Condor, R. et Rebut, K. (2008). Déterminants et modes opératoires du contrôle de gestion dans les PME : une approche qualitative comparative. Actes du Congrès international francophone sur la PME. Louvain-la-Neuve, Belgique, 28-30 mai.

Courrent, J.-M. et Villesèque-Dubus, F. (2008). Recherches en contrôle de gestion et PME : évolution et logiques de structuration. Actes du $29^{e}$ Congrès de l'Association francophone de comptabilité. Bordeaux, France, 29-30 septembre.

Crozier, M. et Friedberg, E. (1977). L’acteur et le système. Paris, Éditions du Seuil.

Davila, T. (2005). An exploratory study on the emergence of management control systems : formalizing human resources in small growing firms. Accounting Organizations and Society, 30(3), 223-248.

Flamholtz, E.G. (1986). How to make the transition from an entrepreneurship to a professionally managed firm. San Francisco, Jossey-Bass Publishers.

Germain, C. (2001). Le pilotage de la performance dans les petites et moyennes entreprises : les résultats d'une recherche empirique. Actes du $22^{e}$ Congrès de l'Association francophone de comptabilité. Toulouse, France, 16-17 mai.

Germain, C. (2004). La contingence des systèmes de mesure de la performance : les résultats d'une recherche empirique sur le secteur des PME. Finance Contrôle Stratégie, 7(1), 33-52.

Germain, C. (2005). Une typologie des tableaux de bord implantés dans les petites et moyennes entreprises. Finance Contrôle Stratégie, 8(3), 125-143.

Haldma, T. et Lä̈̈TS K. (2002). Contingencies influencing the management accounting practices of estonian manufacturing companies. Management Accounting Research, 13(4), 379-400.

Hlady-Rispal, M. (2002). La méthode des cas, application à la recherche en gestion. Bruxelles, De Boeck Supérieur.

JÄNKÄLÄ, S. (2007). Management control systems (MCS) in the small business context : linking effects of contextual factors with MCS and financial performance of small firms. Finlande, Oulu Univ Press.

Julien, P.-A. (2005). Pour une définition des PME. Dans P.-A. Julien (dir.), Les PME : bilan et perspectives (p. 1-30). Canada, Presses Inter Universitaires.

Kaplan, R.S. et Norton, D.P. (2007). L’alignement stratégique. Pairs, Éditions Eyrolles.

LAvigne, B. (2002). Contribution à l'étude de la genèse des systèmes d'information comptable des PME : une approche empirique. Actes du $23^{e}$ Congrès de l'Association francophone de comptabilité. Toulouse, France, 15-17 mai.

Lavigne, B. et St-Pierre, J. (2002). Association entre le système d'information comptable des PME et leur performance financière. Actes $d u 6^{\mathrm{e}}$ Congrès international francophone sur la PME. Montréal, Canada, 30 octobre- $-^{\text {er }}$ novembre.

Macintosh, N.B. (1985). The social software of accounting and information system. Chichester, Royaume-Uni, John Wiley \& Sons.

MacMahon, R.G.P. et Holmes, S. (1991). Small business financial management practices in North America : a literature review. Journal of Small Business Management, 29(2), 19-29. 
Meyssonnier, F. et ZAWADZKi, C. (2008). L'introduction du contrôle de gestion en PME : étude d'un cas de structuration tardive de la gestion d'une entreprise familiale en forte croissance. Revue internationale PME, 21(1), 69-92.

Mintzberg, H. (1982). Structure et dynamique des organisations. Paris, Éditions d'Organisation.

Mintzberg, H. (2006). Le manager au quotidien : les dix rôles du cadre. Paris, Éditions d'Organisation.

Moores, K. et Yuen, S. (2001). Management accounting systems and organizational configuration : a life-cycle perspective. Accounting, Organizations and Society, 26(4), 351-389.

Mucchielli, R. (1988). L’observation psychologique et psychosociologique. Paris, ESF.

NGongang, D. (2007). Analyse des facteurs déterminants du système d'information comptable et des pratiques comptables des PME tchadiennes. Revue des Sciences de Gestion, (2), 49-57.

Nobre, T. (2001). Méthodes et outils du contrôle de gestion dans les PME. Finance Contrôle Stratégie, $4(2), 119-148$.

Nobre, T. et ZaWAdski, C. (2013). Stratégie d'acteurs et processus d'introduction du contrôle de gestion en PME. Comptabilité Contrôle Audit, (1), 91-116.

Oriot, F. et BERgERON, H. (2012). Indicateurs de performance et priorités stratégiques des dirigeants de PME. Dans G. Lecointre (dir.), Le grand livre de l'économie PME (p. 201-225). Paris, Gualino.

Oriot, F. et Bergeron, H. (2015). Simplifier les systèmes de pilotage de la performance des dirigeants de PME : le choix assumé d'une quête de sens et d'action. Dans F. Oriot et H. Bergeron (dir.), Mélanges en l'honneur du Professeur Yves Dupuy (p. 183-190). Paris, Economica.

Oriot, F. et Misiaszek, E. (2012). Le balanced scorecard au filtre d'une PME française : ou pourquoi les PME préfèrent le «sur-mesure ». Revue française de gestion, 38(6), 27-43.

Oriot, F., Otley, D. et Misiaszek, E. (2010). Strategic performance management systems in SME's : exploring new research opportunities. Actes du $31^{e}$ Congrès de l'Association francophone de comptabilité. Nice, France.

RAymond, L. (1995). Les systèmes d'information dans les PME : bilan et perspectives. Paris, Economica.

Raymond, L., Blili, S. et El-Alami, D. (2004). L'écart entre le consultant et la PME : analyse et perspectives. Gestion, 28(4), 52-60.

RAYMOND, L. et BERgERON, F. (2008). Enabling the business strategy of SMEs through e-business capabilities : a strategic alignment perspective. Industrial Management and Data System, 108(5), 577-595.

Rowe, F., Fernandez, V. et Picory, C. (1994). Diversité, cohérence et pertinence des outils de gestion : le cas des PME de l'Île-de-France. Actes $d u$ 39 ${ }^{\mathrm{e}}$ Conférence mondiale des PME. Strasbourg, France, juin.

SABOLY, M. (1994). Les déterminants de la qualité des produits comptables : le rôle du dirigeant (thèse de doctorat en sciences de gestion). Université de Poitiers, France.

Santin, S. et Van Caillie, D. (2008). Le design du système de contrôle de gestion des PME : une quête de stabilité adaptative. Actes $d u 29^{e}$ Congrès de l'Association francophone de comptabilité. Paris, France, 28-30 mai.

St-Pierre, J. et Delisle, S. (2006). An expert diagnosis system for the benchmarking of SME's performance. Benchmarking: An International Journal, 13(1-2), 106-119.

Thiétart, R.A. (2014). Méthodes de recherche en management (4édition). Paris, Dunod. 
Torrès, O. (2000). Du rôle et de l'importance de la proximité dans la spécificité de gestion des PME. Actes $d u 5^{\mathrm{e}}$ Congrès international francophone sur la PME. Lille, 25-27 octobre.

Torrès, O. (2004). Essai de théorisation de la gestion des PME : de la mondialisation à la proxémie (habilitation à diriger des recherches en sciences de gestion). Université de Caen.

Vallerand, J., Berthelot, S. et Morrill, J. (2008). Positionnement de la PME manufacturière canadienne face aux outils de gestion enseignés dans les programmes de formation universitaire en administration. Actes du $9^{e}$ Congrès international francophone sur la PME. Louvain-la-Neuve, Belgique, 29-30-31 octobre.

Van Caillie, D. (2003). L'exercice du contrôle de gestion en contexte PME : étude comparée des cas français, canadien et belge. Actes du $25^{e}$ Congrès de l'Association francophone de comptabilité. Louvain-la-Neuve, Belgique, 22-23 mai.

Whitley, R. (1999). Firms, institutions and management control : the comparative analysis of coordination and control systems. Accounting Organizations and Society, 28(5-6), 507-524.

ZAWADZKI, C. (2011). L'évolution des routines organisationnelles en PME : leçons de l'échec d'une démarche d'introduction du contrôle de gestion. Actes du $32^{\circ}$ Congrès de l'Association francophone de comptabilité. Montpellier, France, 9-10-11 mai. 